NASA

Technical Memorandum 107387
0,0360

Army Research Laboratory

Technical Report ARL-TR-1253

\title{
Endwall Heat Transfer Measurements in a Transonic Turbine Cascade
}

P.W. Giel

NYMA, Inc.

Brook Park, Ohio

D.R. Thurman

U.S. Army Research Laboratory

Lewis Research Center

Cleveland, Ohio

G.J. Van Fossen, S.A. Hippensteele, and R.J. Boyle

Lewis Research Center

Cleveland, Ohio

Prepared for the

41st Gas Turbine and Aeroengine Congress

sponsored by the International Gas Turbine Institute of

the American Society of Mechanical Engineers

Birmingham, United Kingdom, June 10-13, 1996

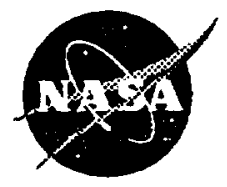

National Aeronautics and

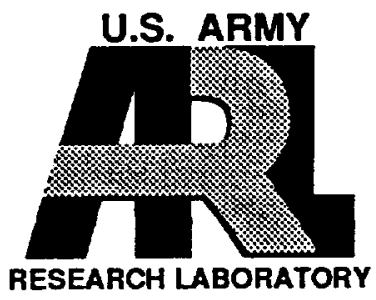




\title{
ENDWALL HEAT TRANSFER MEASUREMENTS IN A TRANSONIC TURBINE CASCADE
}

\author{
P. W. Giel \\ NYMA, Inc. \\ Engineering Services Division \\ Brook Park, OH
}

\author{
D. R. Thurman \\ U.S. Army Research Laboratory \\ NASA Lewis Research Center \\ Cleveland, $\mathrm{OH}$
}

G. J. Van Fossen, S. A. Hippensteele, and R. J. Boyle Internal Fluid Mechanics Division NASA Lewis Research Center Cleveland, $\mathrm{OH}$

\begin{abstract}
Turbine blade endwall heat transfer measurements are given for a range of Reynolds and Mach numbers. Data were obtained for Reynolds numbers based on inlet conditions of 0.5 and $1.0 \times 10^{6}$, for isentropic exit Mach numbers of 1.0 and 1.3, and for freestream turbulence intensities of $0.25 \%$ and $7.0 \%$. Tests were conducted in a linear cascade at the NASA Lewis Transonic Turbine Blade Cascade Facility. The test article was a turbine rotor with $136^{\circ}$ of turning and an axial chord of $12.7 \mathrm{~cm}$. The large scale allowed for very detailed measurements of both flow field and surface phenomena. The intent of the work is to provide benchmark quality data for CFD code and model verification. The flow field in the cascade is highly three-dimensional as a result of thick boundary layers at the test section inlet. Endwall heat transfer data were obtained using a steady-state liquid crystal technique.
\end{abstract}

\section{LIST OF SYMBOLS}

$A \quad$ - heater area $\left[\mathrm{cm}^{2}\right]$

$C_{p} \quad-$ specific heat $[\mathrm{J} / \mathrm{kg} \cdot \mathrm{K}]$

$C_{x}$ - blade axial chord $[\mathrm{cm}]$

$E c$ - Eckert number, $E c=U^{2} /\left(C_{p} \Delta T\right)$

$h$ - heat transfer coefficient $\left[\mathrm{W} / \mathrm{m}^{2} \cdot \mathrm{K}\right]$

$k$ - thermal conductivity [W/m.K]

$\begin{array}{lll}I & - & \text { electrical current [Amps] } \\ L & - & \text { heater length in y-direction [cm] } \\ L_{x} & - & \text { longitudinal integral turbulence length } \\ & & \text { scale [cm] } \\ \dot{m} & - & \text { cascade mass flow rate }[\mathrm{kg} / \mathrm{s}] \\ M & - & \text { Mach number } \\ p & - & \text { blade pitch [cm] } \\ P & - & \text { pressure [Pa] } \\ P r & - & \text { Prandtl number } \\ q & - & \text { heat transfer rate [Watts] } \\ r & - & \text { recovery factor, } r=\mathrm{Pr}^{1 / 3} \\ \mathcal{R} & - & \text { heater resistance [Ohms] } \\ R & - \text { gas constant [J/kg.K] } \\ R e_{C_{x}} & - \text { Reynolds number, } \operatorname{Re}_{C_{x}}=\rho U_{i n} C_{x} / \mu \\ s & - \text { blade span [cm] } \\ S t & - \text { Stanton number } \\ t & - \text { thickness [cm] } \\ T & - \text { temperature [K] } \\ T u & - \text { Turbulence intensity } \\ U & - \text { total velocity [m/s] } \\ V & - \text { electrical voltage [Volts] } \\ x & - \text { chordwise (axial) direction } \\ y & - \text { pitchwise (tangential) direction } \\ z & - \text { spanwise (radial) direction } \\ \alpha & - \text { pitch angle }(x-y) \text { plane [deg.] } \\ \gamma & - \text { specific heat ratio, } \gamma=1.4 \\ & \end{array}$




\begin{tabular}{|c|c|c|}
\hline$\delta$ & - & $99 \%$ boundary layer thickness $[\mathrm{cm}]$ \\
\hline$\epsilon$ & - & emissivity \\
\hline$\mu$ & - & dynamic viscosity $[\mathrm{kg} / \mathrm{s} \cdot \mathrm{m}]$ \\
\hline$\rho$ & - & density $\left[\mathrm{kg} / \mathrm{m}^{3}\right]$ \\
\hline$\sigma$ & - & Stefan-Boltzmann constant \\
\hline Subscripts & & \\
\hline$a w$ & - & adiabatic wall temperature \\
\hline bulk & - & bulk heat addition \\
\hline le & - & liquid crystal \\
\hline ex & - & exit freestream value \\
\hline in & - & inlet freestream value \\
\hline IS & - & isentropic value \\
\hline htr & - & heater \\
\hline ref & - & $\begin{array}{l}\text { evaluated at Eckert's reference } \\
\text { temperature }\end{array}$ \\
\hline Superscripts & - & surface \\
\hline ' & - & total conditions \\
\hline
\end{tabular}

\section{INTRODUCTION}

As the dependence on computational analyses for aerodynamics and heat transfer increases in the design of turbomachinery, so does the need for detailed, benchmark quality experimental data. These data are required for validation of existing turbomachinery CFD analyses, and to provide data for improved approaches to modeling the flow field characteristics. Because of the sensitivity of the life of high pressure turbine rotors to metal temperature, validation of the procedures used to predict these temperatures is essential. Validation of CFD analyses should be done at conditions which approach engine operating conditions as closely as possible. Several experiments have obtained detailed data for turbine rotor geometries. Langston et al. (1977), Gregory-Smith and Graves (1983), Marchal and Sieverding (1977), Yamamoto (1987a,b), and Gregory-Smith et al. (1988) presented detailed results of aerodynamic measurements for rotor blades, tested in linear cascades at relatively low Mach numbers. Mee et al. (1992a) and Mee et al. (1992b) presented measurements for a rotor linear cascade tested at transonic conditions obtained in a short duration blowdown facility. Heat transfer measurements for turbine rotor geometries were obtained by Graziani et al. (1980), Goldstein and Spores (1988) in linear cascades, and by Blair (1994) in a rotating cascade. All of the heat transfer experiments were done in large scale test facilities, but were at low Mach number. To increase confidence in the ability of computational analyses to predict turbine rotor heat transfer under actual engine conditions, there exits a need to obtain both aerodynamic and heat transfer data at high Mach number conditions.
A Transonic Turbine Blade Cascade Facility was built at the NASA-Lewis Research Center to provide detailed aerodynamic and heat transfer measurements at high Mach numbers. The facility is a linear cascade. It was recognized that a linear cascade cannot simulate rotation effects, and that the chosen design does not allow for tip clearance effects. Nonetheless, a linear cascade was chosen over an annular cascade for a number of reasons. The primary reason was to obtain detailed measurements in a large scale facility at transonic flow conditions. The physical size of the blading was determined by the amount of air that could be moved at transonic speed through the cascade. An annular cascade of engine typical hub-to-shroud and aspect ratios would require several times more flow rate than a linear cascade for blades of the same chord length. Also, a linear cascade provides easier access for instrumentation than an annular cascade. From a computational aspect, a linear geometry minimizes the effects of the three-dimensional mesh on the CFD solution, thus giving a clearer analysis of the actual flow solver.

Some noteworthy features of the linear cascade facility are: its ability to achieve transonic Mach numbers, its high blade count to ensure good periodicity, and its large scale. The large scale facilitates highly localized and detailed flow and heat transfer measurements. Because of the large scale $\left(C_{x}=12.7 \mathrm{~cm}\right)$, and transonic flow conditions, inlet Reynolds numbers up to one million can be achieved with an inlet pressure near one atmosphere. The purpose of the research in this facility is to obtain a detailed aerodynamic and heat transfer database for Reynolds and Mach numbers relevant to high pressure turbine applications. The results of the investigation are available for CFD code and model validation.

The rotor tested in the cascade is of constant cross section, so that the geometry of the cascade is twodimensional. It was desired to provide verification data for three-dimensional CFD analyses. The three dimensionality of the flow field is achieved by not bleeding off the endwall boundary layers. Giel et al. (1996) show that when the endwall boundary layers are not bled off, the spanwise variation of surface pressure on the rotor was of the same order as the spanwise variation in surface pressure experienced in an actual engine. For some tests a square bar grid was placed upstream of the blade row to generate high levels of freestream turbulence. Without the turbulence grid present, the measured inlet freestream turbulence was about $0.25 \%$. With the grid installed, the freestream turbulence was in excess of $7 \%$. 
Endwall heat transfer data was obtained using a steady-state liquid crystal technique. The liquid crystal heat transfer measurement technique was chosen because of the good spatial resolution that it offers. Several versions of the technique have been used for gas turbine related measurements. A transient technique was used by Martinez-Botas et al. (1994) while a steadystate technique was used by Hippensteele and Russell (1988).

The objective of the current paper is to present the results of endwall heat transfer measurements that were made in the Transonic Turbine Blade Cascade. Data were obtained for eight different flow conditions. The results from the eight cases will be presented to illustrate the effects of varying Reynolds number, exit Mach number, and the inlet turbulence grid. Heat transfer measurements were made at inlet Reynolds numbers of 0.5 and $1.0 \times 10^{6}$. Tests were made at pressure ratios corresponding to exit isentropic Mach numbers of 1.0 and 1.3. Tests were conducted at high and low inlet turbulence levels.

\section{DESCRIPTION OF FACILITY}

The initial description of the facility was given by Verhoff et al. (1992). Subsequently, the cascade inlet section was analyzed and redesigned (Giel et al. 1994) to improve the inlet flow pitchwise uniformity. Figure 1 shows an overall view of the facility, including the redesigned forward inlet boards.

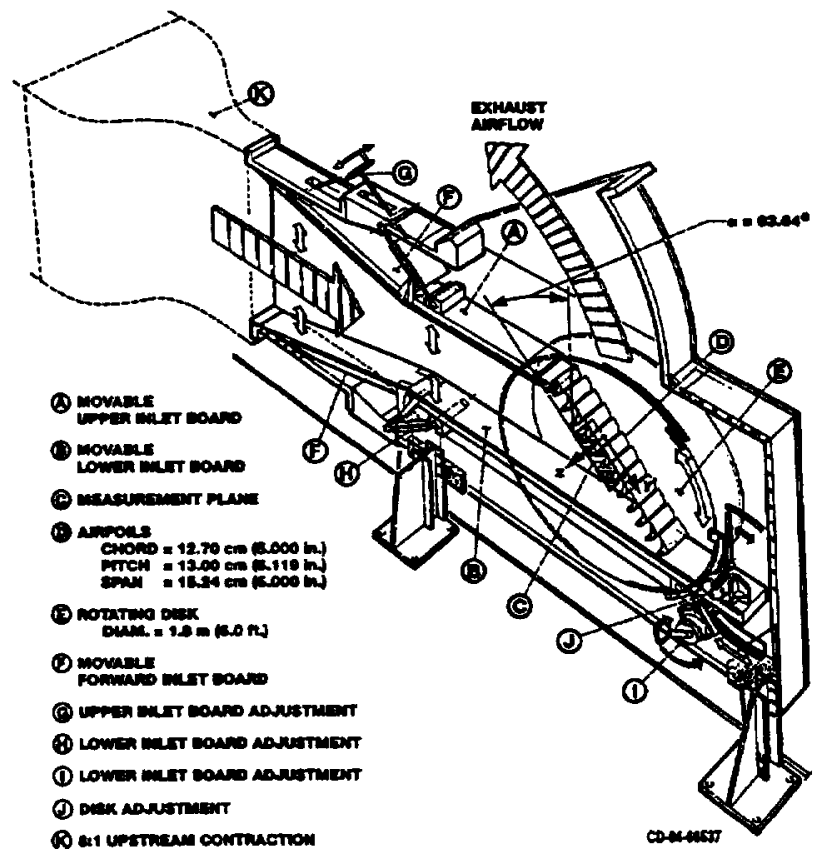

Fig. 1 Overall view of Transonic Turbine Blade Cascade test section
High pressure air at ambient temperature is throttled to give a maximum total pressure of approximately one atmosphere at the inlet to the test section. The high Reynolds number tests had an inlet total pressure near one atmosphere, and the low Reynolds number tests had an inlet total pressure near one-half atmosphere. The air was discharged into an exhaust header maintained at a nominal pressure of $15.9 \mathrm{kPa}$ (2.3 psia). A valve between the test section and exhaust header was used to throttle the flow to establish the desired exit Mach number. As seen in Fig. 1, the test section is mounted on a large disk. This disk can be rotated to give a range of incidences. All of the results described were obtained at the design inlet flow angle of $63.6 \mathrm{de}-$ grees. This inlet angle gave a flow turning of about 136 degrees. Upstream inlet boards were installed as described in Giel et al. (1994), but to prevent shock reflections in the downstream section, no exit tailboards were used. A highly three-dimensional flow field was obtained in the blade passages by allowing boundary layers to develop in the long inlet section upstream of the cascade. Aerodynamic probe data and blade loading data both verify the existence of strongly threedimensional passage flow.

The blade shape used in these tests, while generic in nature, was designed to simulate the flow characteristics of a high specific work rotor. Pertinent details of the blade and of the cascade are given in Table 1. A plan view of the test section is shown in Fig. 2. The figure shows the five $0.025 \mathrm{~mm}$ (0.001 inch) thick Inconel heater sheets and their positions relative to the blades. Passage 5 is the primary measurement passage. The heated section begins upstream of the blade leading edge plane at $-0.3 C_{x}$ and extends downstream to $2.1 C_{x}$. Each of the heaters are $L=2.1 C_{x}$ wide in the $y$-direction. The figure also shows the outline of the $6.35 \mathrm{~cm}(2.50$ in.) thick plexiglas window that was used to gain optical access to the test section. The window formed the endwall opposite the instrumented endwall. The camera fields of view are also shown in the figure. Camera 1 was used primarily to obtain data in the blade leading edge region while Camera 2 was used primarily in the passage and trailing edge regions. Camera 3 had a wider angle lens which allowed a view of the entire test section. It was thus used to obtain data in the far downstream region, as well as in the upstream region near the heater leading edge. Camera 1's view of this region was shadowed by the frame holding the plexiglas window. All of the cameras were located $2.0 \mathrm{~m}$ from the instrumented endwall. Cameras 1 and 2 had $135 \mathrm{~mm}$ focal length lenses while Camera 3 had an $85 \mathrm{~mm}$ focal length lens. 
Table 1 Blade and cascade parameters and dimensions

\begin{tabular}{|l|l|}
\hline Geometric parameter & Value \\
\hline axial chord & $12.70 \mathrm{~cm}(5.000$ inches $)$ \\
pitch & $13.00 \mathrm{~cm}(5.119$ inches $)$ \\
span & $15.24 \mathrm{~cm}(6.000$ inches $)$ \\
true chord & $18.42 \mathrm{~cm}(7.250$ inches $)$ \\
stagger angle & $41.54^{\circ}$ \\
throat diameter & $3.358 \mathrm{~cm}^{2}(1.393$ inches $)$ \\
throat area: 1 passage & $53.94 \mathrm{~cm}^{2}\left(8.360\right.$ in $\left.^{2}\right)$ \\
leading edge diameter & $2.657 \mathrm{~cm}(1.046$ inches $)$ \\
trailing edge diameter & $0.518 \mathrm{~cm}(0.204$ inches $)$ \\
turbulence grid & $2.54 \mathrm{~cm}$ square bar \\
\hline Flow parameter & Value \\
\hline Inlet $R e_{C_{s}}$ & $1.0 \times 10^{6}$ or $0.5 \times 10^{6}$ \\
Exit $R e_{C_{s}}$ & $1.8 \times 10^{6}$ or $0.9 \times 10^{6}$ \\
Inlet $M_{I S}$ & 0.38 \\
Exit $M_{I S}$ & 1.32 or 0.97 \\
Inlet $\delta_{f}$ - no grid & $3.2 \mathrm{~cm}(1.2$ inch $)$ \\
\multicolumn{1}{|c|}{ with grid } & $2.0 \mathrm{~cm}(0.8$ inch $)$ \\
Inlet flow angle & $63.6^{\circ}$ \\
Design flow turning & $136^{\circ}$ \\
\hline
\end{tabular}

The outer wall of the instrumented test section was $3.8 \mathrm{~cm}$ (1.5 in.). thick stainless steel to withstand a pressure difference of one atmosphere. There was $2.3 \mathrm{~cm}\left(0.90 \mathrm{in}\right.$.) of low thermal conductivity $\left(k_{\text {foam }}=\right.$ $0.029 \mathrm{~W} / \mathrm{mK}$ ), high temperature, closed cell foam between the outer wall and the heaters. The foam was coated with $0.3 \mathrm{~cm}(0.10 \mathrm{in}$.) of gelcoat epoxy to provide a smooth surface. "T"-shaped electrical bus bars extended through the endwall assembly. They were flush with the endwall and passed through the outer wall. The inner ends of the bus bars are evident in Fig. 2 at both ends of all five heaters. The Inconel foil was tack welded to the bus bars along these ends. The bus bars were thermally as well as electrically conductive, and were therefore placed outside the test passage. The rest of the foil was bonded to the gelcoat surface using double-sided adhesive tape. The heaters were connected in series outside the test section.

The endwall heater assembly was first painted with flat black lacquer paint and then a uniform grid of white dots were painted on with a template. The dots were used in the data reduction process to determine spatial location. The locations of the reference dots are shown in Fig. 2.

Because the blades were made of stainless steel, those in contact with the heaters needed to have electrically insulated ends. RTV silicone was chosen to coat the ends of the blades. A thickness of $0.4 \mathrm{~mm}(1 / 64 \mathrm{in}$.) was determined from a heat conduction analysis to best balance the insulating effect of the blade, which would tend to allow overheating, and the fin cooling effect of the blade, which would tend to overcool the endwall region near the blade.

The optional $74 \%$ open area square bar turbulence grid is also shown in Fig. 2. The grid consisted of one square $25 \mathrm{~mm} \times 25 \mathrm{~mm}$ ( 1 in. $\times 1$ in.) pitchwise bar that extended between the inlet boards at midspan and three square spanwise bars located on $150 \mathrm{~mm}$ centers.

\section{MEASUREMENT TECHNIQUES}

Inlet Flow Measurements As shown in Fig. 2, aerodynamic probe measurements were made on a plane one axial chord upstream of the blade leading edge plane. The measurements covered three blade pitches and extended from near the endwall, $z / s=8.3 \times 10^{-4}$, to just above midspan, $z / s=0.54$. Details of the measurement techniques are given by Giel et al. (1996). Time mean flow measurements were made with a calibrated 3-hole boundary-layer probe near the endwall and a calibrated 5-hole pitch-yaw probe away from the endwall. Turbulence measurements were made with a constant temperature hot wire anemometer.

Endwall Static Pressure Measurements The local isentropic Mach number is needed to calculate the endwall surface Stanton number distribution. Surface static pressure data was obtained with a separate endwall, instrumented only with pressure taps. The test passage and two adjacent passages were each instrumented with 87 pressure taps per passage. The taps extended axially from $x / C_{x}=-0.4$ to $x / C_{x}=2.1$, and were arranged in 21 rows with either 4 or 5 taps per row. Pressure measurement taps were placed in all passages, but away from the test passage fewer measurements were made. The locations of the taps will be shown along with the data in a following section. More details of the endwall static pressure measurement techniques are given by Giel et al. (1996).

Heat Transfer Measurements The steady-state liquid crystal technique was chosen for the present study for several reasons. Primarily, the method is relatively insensitive to substrate non-homogeneity (e.g. air bubbles, thickness variations, etc.). Furthermore, the isotherms can be photographed with higher quality still photographs rather than with video that has poorer spatial and color resolution. The following is a brief overview of the technique: Thin Inconel sheets were placed on the endwall so as to cover the surface of the test blade passage. A suspension of liquid crystals was painted on the Inconel sheets. Electrical power was 


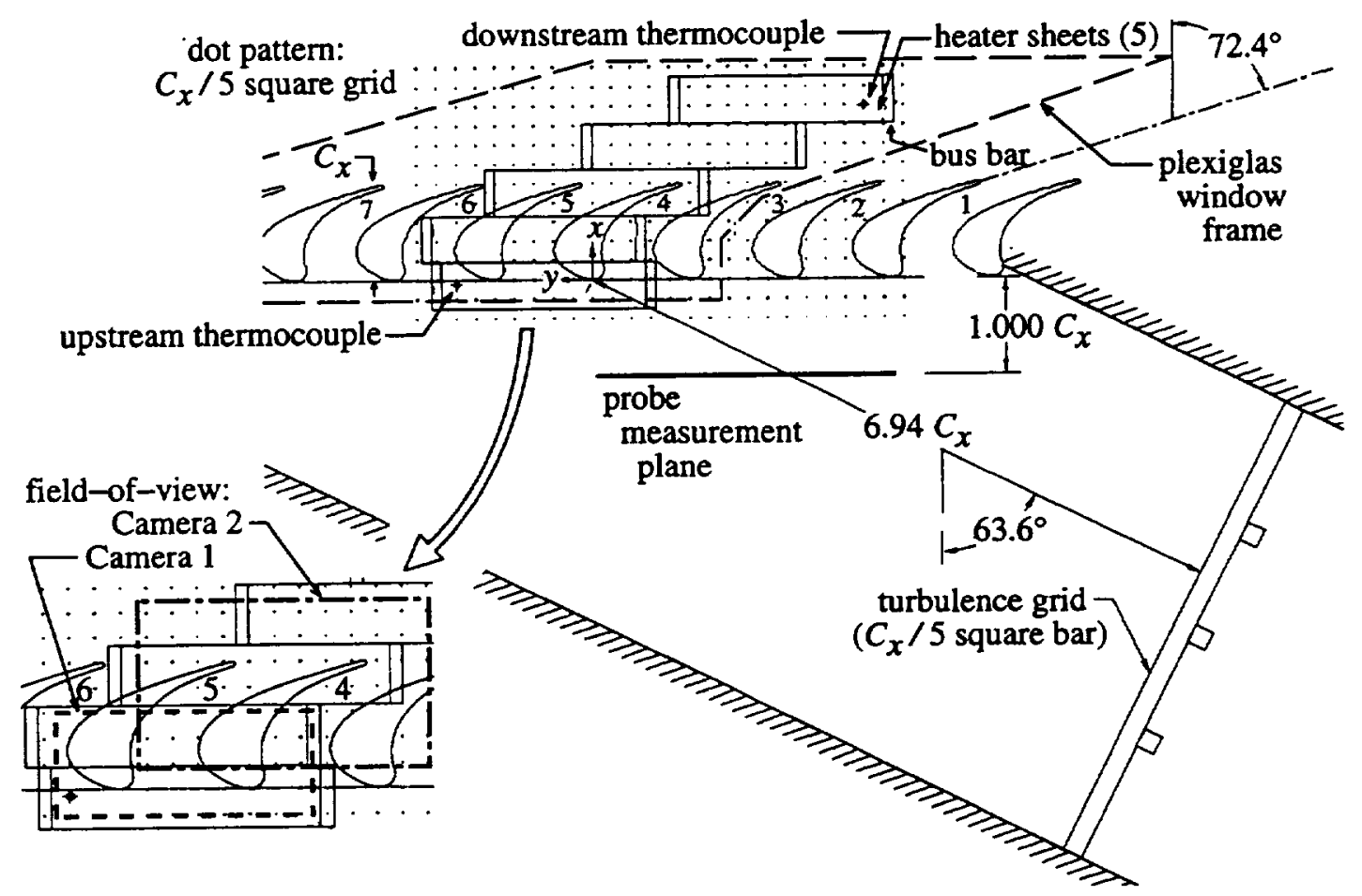

Fig. 2 Geometry of endwall test section

supplied to the Inconel sheets to generate a spatially uniform heat flux. For each liquid crystal, there is a very narrow temperature band at which a well-defined color is visible. Where the color is observed, the surface temperature is known. Since the electrical power is known, the heat transfer coefficients can be determined. Varying the electric power changes the location of the liquid crystal isotherm. When steady-state conditions were achieved, the locations of the isotherms were photographically recorded. Finally, the locations of the isotherms, along with endwall isentropic Mach number distributions, were used to generate contours of Stanton number. Details of all of the steps in this process will now be discussed.

Two micro-encapsulated chiral nematic liquid crystals with yellow-band temperatures of $T_{i c, 1}=37.9 \pm 2^{\circ} \mathrm{C}$ $\left(100.2 \pm 3.6^{\circ} \mathrm{F}\right)$ and $T_{l c, 2}=64.5 \pm 3^{\circ} \mathrm{C}\left(148.1 \pm 5.4^{\circ} \mathrm{F}\right)$ were mixed and sprayed onto the surface. The crystal temperatures were calibrated using the surfacemounted thermocouples shown in Fig. 2. As described by Moffat (1990), micro-encapsulated crystals tend to be less sensitive to illuminating and viewing angles than non-encapsulated crystals. While the optical axes of all three cameras were orthogonal to the planar endwall, strobe lights used for illumination were mounted at various angles. To best illuminate the views, two photographs were taken with each of the three cameras, one with a strobe to the right of the camera and one with a strobe to the left. No differences in isotherm location were ever detected between the two photographs.

The voltage drop, $V$, across each of the five heaters was measured independently. The current, $I$, through the entire circuit was measured with three redundant $0.1 \%$ accuracy shunts. From these voltage and current measurements, the power, $q_{h t r}=V I$, and the resistance, $\mathcal{R}=V / I$, were calculated, displayed, and recorded.

One disadvantage of the steady-state liquid crystal method is that longer run times are required for thermal equilibration to be achieved. After changing the heater power level, the steady-state condition was examined by monitoring the isotherm position on a remote video display. The resistance of the heaters was also monitored. Inconel was chosen as the heater material because of its low coefficient of thermal resistivity. The signals giving the heater voltage and current had high signal-to-noise ratios. Even though the calculated resistance varied by less than $0.5 \%$ over the entire range of heater power settings, variations in resistance with temperature could be monitored. Stability of the resistance was also used as an indicator of when steady state conditions were achieved. 
When steady-state conditions were achieved, $35 \mathrm{~mm}$ color slide photographs were taken of the endwall surface with three cameras. Each camera was positioned to view a subset of the entire surface, with some overlap between camera views as described previously and as shown in Fig. 2. The endwall was illuminated using high speed strobe lights. The short duration of the strobe light prevented radiative heating of the liquid crystals. Two strobes were used for each camera, and were positioned to prevent blind spots caused by reflections or shadows. Pressures, temperatures, and other data were recorded simultaneously with the photographic record. The resulting photographic slides were then projected onto paper marked with the dot pattern shown in Fig. 2. The dot pattern in the slide image was aligned with the dot pattern on the paper. Composite drawings of the yellow line positions were made. Each camera and crystal temperature had a separate drawing. Flow condition data, time, crystal temperature, and power level were associated with each line. These lines were then digitized. The coordinates and their associated data were stored for further processing. Typically, about 5000 digitized points were stored for each flow condition run. A sample of the digitized point distribution is shown in Fig. 3. The full range of surface Stanton numbers was typically obtained from 25 different power levels. Typical power settings ranged from 200 to 1200 Watts in increments of 15 to 100 Watts. The particular power settings used depended on the inlet ambient temperature.

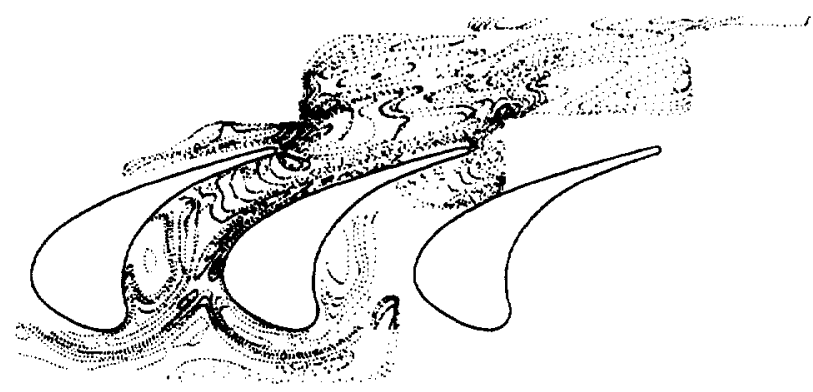

Fig. 3 Sample digitized data point distribution

The coordinates of each digitized point, along with the data associated with the point, were read into a data reduction program. The purpose of the program was to calculate Stanton numbers at each digitized point, but this calculation required knowledge of the local adiabatic wall temperature. The first step in the procedure was thus to read in CFD calculations of endwall surface static pressure, which will later be shown to agree well with the experimental data. The calculated values provided significantly better spatial resolution than the experimental pressure distribution. Isentropic sur- face Mach numbers were determined at each digitized point by interpolation from the CFD calculations. After determining $M_{I S}, T_{a w}$ and thus the Stanton number could be determined at each of the digitized points. The Stanton and Mach numbers were then interpolated onto a uniform, rectangular grid to generate contour plots. For all eight flow conditions, a $176 \times 41$ grid was used on each heater. Three point linear interpolation was used, and care was taken never to interpolate across the blade boundaries. Since the Mach numbers originated as calculated values, they had extremely fine spatial resolution. Contour plots of the interpolated Mach numbers were also created for verification. Comparing contour plots of the original, input Mach number distribution with contour plots generated after the Mach numbers had been first interpolated onto each digitized point, and then interpolated onto the rectangular grid, validated the interpolation procedure. If the two contour plots did not agree, it was determined that this was a region of insufficient data in which no Stanton number contours should be shown. For all of the test cases this situation arose only near the edges of the measurement area.

The range of electric power supplied to the endwall heaters was limited so that they became neither too hot nor too cool. The heaters were assembled in a highly stretched condition at room temperature. At low temperatures the thin heaters could stretch beyond their elastic limit, and then wrinkle when warmed. The heaters could become too cool at low power levels, especially near the blade row exit, because of the low adiabatic wall temperatures associated with high Mach numbers. At high power levels, and thus at high surface temperatures, the adhesive ability of the doublefaced tape is reduced and the heaters could unbond from the surface. The choice of crystal temperatures, $37.9^{\circ} \mathrm{C}\left(100.2^{\circ} \mathrm{F}\right)$, or $64.5^{\circ} \mathrm{C}\left(148.1^{\circ} \mathrm{F}\right)$, was made with consideration to these high and low power limits.

\section{Definition of the Heat Transfer Coefficient}

While the liquid crystal technique offers the advantage of good spatial resolution, this technique does not facilitate heat transfer measurements at a fixed point for varying power levels. Measurements of this type can be obtained using surface mounted thermocouples. In the present study, two thin film thermocouples were bonded to the Inconel heaters, one upstream of the blade row and one downstream (see Fig. 2). Measurements from these thermocouples were used to determine the most appropriate definition of heat transfer coefficient. The thin layers of paint and liquid crystals influenced the thermocouple measurements, but this data was used only to compare Stanton number definitions. Adding 
corrections for the paint conductance simply shifts the level of $S t$, but the trends that will now be described remain the same. Even for relatively small temperature differences such as in these tests, the heat transfer coefficient should be independent of the heat flux level. Because of the low temperature differences and the high Mach numbers, the local Eckert numbers were very high. Consequently, the choice of temperature difference used as the driving potential has a significant effect on the heat transfer coefficient. The most appropriate definition of Stanton number is the one which results in values that do not depend on the level of surface heat flux. In general, the definitions of the heat transfer coefficient, $h$, and of the Stanton number, $S t$, are:

$$
S t=\frac{q / A}{\rho U_{i n} C_{p} \Delta T}=\frac{h}{\rho U_{\text {in }} C_{p}}
$$

Each of the terms in this equation can be defined or evaluated at different locations or at different temperatures. In the following discussion, the downstream thermocouple readings were examined to determine an appropriate set of terms for use in calculating the Stanton number. These results are for the case of an inlet $\operatorname{Re}_{C_{x}}=0.973 \times 10^{6}, M_{\text {ex }}=1.328$, and with the turbulence grid installed. This case provides the most contrast between definitions, but the trends are consistent for both thermocouples and for all flow cases. Five different definitions of Stanton number are described in Table 2, and the results of those definitions are shown in Fig. 4. For all definitions the reference velocity was chosen as $U_{\text {in }}$ and $C_{p}$ was assumed to be constant. Also for all of the definitions, $q$ represents the net surface heat transfer rate. The density used in the Stanton number calculations, unlike the value used for the Reynolds number, is not the actual physical density. For definitions $A$ and $B, \rho$ is the inlet total density. For other definitions it is calculated using inlet total pressure and a temperature as shown in Table 2. The local adiabatic wall temperature, $T_{a w}$, is defined as:

$$
\frac{T_{a w}}{T_{i n}^{\prime}}=r+\frac{1-r}{1+0.5(\gamma-1) M^{2}}
$$

The isentropic Mach number, $M$, was determined from endwall static pressure measurements. A reference temperature for properties given by Eckert (1955) also appears in Table 2. Figure 4 clearly shows that either definition $D$ or $E$ is the most appropriate for the current study. Both of the definitions unfortunately require an iterative calculation because of the interdependency between $T_{a w}$ and $T_{\text {ref }}$. Definition $E$ accounts for the bulk temperature rise caused by the endwall heaters and in general would therefore be more appropriate than $D$.
For the relatively high Reynolds numbers and small temperature differences of the current study, bulk heating has little effect on $S t$, but at lower Reynolds numbers or larger temperature differences this may not be the case. Accounting for the effects of bulk heating requires an integration of the form $\int q d A$ which is quite simple for a uniform $q$ boundary condition, but which is more complex if the flow is modeled with a uniform wall temperature boundary condition. Since many CFD codes implement the simpler uniform wall temperature boundary condition, all data presented here will be based on definition $D$.

Figure 4 also shows an overall variation in $S t$ of about $5 \%$ for either definition $D$ or $E$. As will be described, the overall uncertainty of the liquid crystal measurements was estimated to be $6 \%$ to $11 \%$. The primary reason for the high sensitivity of the Stanton number to the choice of temperatures is that local Eckert numbers are high. At the blade row exit, and at a temperature corresponding to the lower temperature liquid crystal, the local Eckert number was approximately 8. At these relatively high Eckert numbers, heat transfer rates are a function of both Reynolds number and Mach number. The careful definition of Stanton number described above properly accounts for the Mach number dependency through the use of the adiabatic wall temperature and Eckert's reference temperature. The reference temperature accounts for thermophysical property variations. It is interesting to note that the thermocouple temperature matched $T_{i c, 1}$ at $q_{h t r}=560$ Watts and matched $T_{l c, 2}$ at $q_{h t r}=1300$ Watts.

The net surface heat transfer rate, $q$, used to calculate the Stanton number, is the heater power corrected for back-face conduction losses and for radiative heat transfer to other surfaces:

$q=q_{h t r}-k_{\text {foam }} A\left(T_{s}-T_{\text {foam }}\right) / t_{\text {foam }}-\epsilon \sigma A\left(T_{s}^{4}-T_{a w}^{4}\right)$

where $q_{h t r}=V I$ and $A$ is the total heater area $\left(824.2 \mathrm{~cm}^{2}\right)$. The second term in this equation represents the back-face heat conduction loss, and was less than $0.6 \%$ of $q_{h t r}$. The third term represents the radiative losses, and was less than $4.5 \%$ of $q_{h t r}$. For the results shown in Fig. 4, $T$, was measured with the surface thermocouple. When endwall Stanton numbers were being determined, $T$, was the temperature of the liquid crystal. $T_{\text {foam }}$ was measured on the back face of the insulating foam, $k_{\text {foam }}$ and $t_{\text {foam }}$ were given above, and $\epsilon=0.98$. 
Table 2 Definitions used for Stanton number terms

$$
S t=\frac{q / A}{\rho U_{\text {in }} C_{p}\left(T_{s}-T_{g}\right)}
$$

\begin{tabular}{|c|c|c|}
\hline Def'n & $\rho$ & $T_{g}$ definition \\
\hline A & $\frac{P_{i n}^{\prime}}{R T_{i n}^{\prime}}$ & $T_{i n}^{\prime}$ \\
\hline B & $\frac{P_{i n}^{\prime}}{R T_{i n}^{\prime}}$ & $\begin{array}{c}T_{a w}=T_{i n}^{\prime}\left[r+\frac{1-r}{1+0.5(\gamma-1) M^{2}}\right] \\
r=\operatorname{Pr}^{1 / 3}, \operatorname{Pr}=\operatorname{Pr}\left(T_{e x}\right)\end{array}$ \\
\hline $\mathrm{C}$ & $\frac{P_{i n}^{\prime}}{R T_{r e s}}$ & $\begin{array}{c}T_{a w}=T_{i n}^{\prime}\left[r+\frac{1-r}{1+0.5(\gamma-1) M^{2}}\right] \\
T_{r e f}=T+0.5(T,-T)+ \\
0.22\left(T_{a w}-T\right) \\
r=\operatorname{Pr}^{1 / 3}, \operatorname{Pr}=\operatorname{Pr}\left(T_{e x}\right)\end{array}$ \\
\hline D & $\frac{P_{i n}^{\prime}}{R T_{r e s}}$ & $\begin{array}{c}T_{a w}=T_{i n}^{\prime}\left[r+\frac{1-r}{1+0.5(\gamma-1) M^{2}}\right] \\
T_{r e f}=T+0.5\left(T_{s}-T\right)+ \\
0.22\left(T_{a w}-T\right) \\
r=\operatorname{Pr}^{1 / 3}, \operatorname{Pr}=\operatorname{Pr}\left(T_{\text {ref }}\right)\end{array}$ \\
\hline $\mathrm{E}$ & $\frac{P_{i n}^{\prime}}{R T_{r e s}}$ & $\begin{array}{r}T_{a w}=\left(T_{\text {in }}^{\prime}+\Delta T_{\text {bulk }}\right) \times \\
{\left[r+\frac{1-r}{1+0.5(\gamma-1) M^{2}}\right]} \\
T_{r e f}=T+0.5\left(T_{s}-T\right)+ \\
0.22\left(T_{a w}-T\right) \\
r=P r r^{1 / 3} \operatorname{Pr}=P r\left(T_{\text {ref }}\right) \\
\Delta T_{b u l k}=\frac{q_{h t r}\left(x / C_{x}+0.3\right) L_{h t r} / A}{\frac{0.5 \dot{m} L_{h t r} C_{p}}{11 p}}\end{array}$ \\
\hline
\end{tabular}

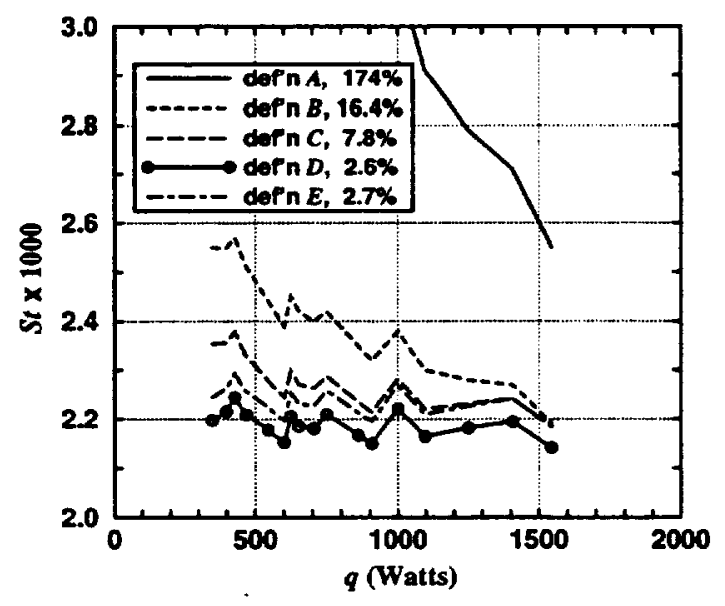

note: $\%$ variations defined as $\frac{S t @ q_{\min }-S t @ q_{\max }}{S t @ q_{\max }} \times 100 \%$

Fig. 4 A Comparison of Stanton number definitions
An uncertainty analysis was performed following the method of Kline and McClintock (1953). The analysis showed that the $\Delta T$ term in the calculation of Stanton number was the major source of uncertainty. Based on the following uncertainties: $T_{l c, 1}= \pm 0.5^{\circ} \mathrm{C}$ $\left( \pm 0.9^{\circ} \mathrm{F}\right), T_{l c, 2}= \pm 0.8^{\circ} \mathrm{C}\left( \pm 1.4^{\circ} \mathrm{F}\right)$, and $T_{a w}= \pm 0.6^{\circ} \mathrm{C}$ $\left( \pm 1.0^{\circ} \mathrm{F}\right)$, the overall uncertainty in $S t$ was determined to be less than $11 \%$. The liquid crystal uncertainties were estimated by applying power to the heaters and monitoring the surface mounted thermocouples. The crystals were viewed using the same photographic techniques that were used during actual data acquisition. To minimize conduction errors through the thin paint layer between the thermocouple and the liquid crystals, air flow was turned off and the pressure in the test section was reduced to near vacuum, thus minimizing natural convection. During the actual data acquisition, the high temperature liquid crystal data were used if the temperature difference, $\Delta T$, using the low temperature liquid crystal was too low to maintain the desired maximum uncertainty in Stanton number.

\section{MEASUREMENT RESULTS}

Inlet Flow Measurements Well-documented inlet boundary conditions are needed for CFD calculations. Figure 5 shows the results of aerodynamic inlet measurements on a plane one axial chord upstream of the blade leading edge plane. Figures $5 \mathrm{a}$ and $5 \mathrm{~b}$ show local Mach number contours without a turbulence grid and with the turbulence grid installed. Both figures present data obtained at $\operatorname{Re}_{C_{x}}=1.0 \times 10^{6}$. Based on flat plate boundary layer correlations, the $R e_{C_{x}}=0.5 \times 10^{6}$ cases can approximately be expected to have a $10 \%$ thicker inlet boundary layer. The exit Mach number for the endwall heat transfer cases was either 1.0 or 1.3 leaving the flow choked at the throat and thus leaving the inlet flow independent of $M_{e x}$.

Figure 5 shows that without the turbulence grid there is a thick and pitchwise periodic inlet boundary layer. Because of the large leading edge diameter, the effects of the blades are evident even one axial chord upstream of the leading edge. Comparing Figs. 5a and $5 \mathrm{~b}$ shows that the presence of the grid adversely affects the flow uniformity. The primary measurement passage, Passage 5, has a midrange level of turbulence intensity, but a higher than average velocity. In terms of Reynolds number, $R_{C_{x}}$ in Passage 6 was reduced about $8 \%$ while $\operatorname{Re}_{C_{x}}$ in Passage 4 remained about the same. Without a grid, the inlet $T u$ was uniform at about $0.25 \%$ for $\operatorname{Re}_{C_{x}}=1.0 \times 10^{6}$ and $0.50 \%$ at $\operatorname{Re}_{C_{x}}=0.5 \times 10^{6}$. Figure $5 \mathrm{c}$ shows the turbulence intensity distribution with the grid installed. The turbu- 
lence intensity, $T u$, in Fig. $5 \mathrm{c}$ has been normalized by the spanwise and pitchwise averaged inlet velocity. The roughly linear dependence of $T u$ on pitchwise distance resulted from the grid being installed perpendicular to the mean flow, thus making it not parallel to the measurement plane and the blade leading edge plane. The measurement and blade leading edge planes were parallel. Gregory-Smith and Cleak (1992) showed that a $42.75^{\circ}$ skewed grid produced a mean flow deflection of more than $1.0^{\circ}$. Skewing the grid in the present study may have resulted in a more uniform $T u$ distribution, but it probably would have had an even more detrimental effect on the mean velocity. The spanwise and pitchwise averaged inlet velocity was used to calculate $R e_{C_{x}}$ and $S t$.

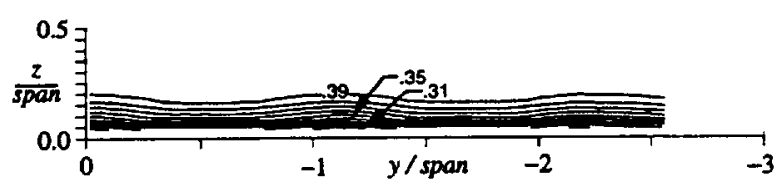

a. Mach number distribution without turbulence grid

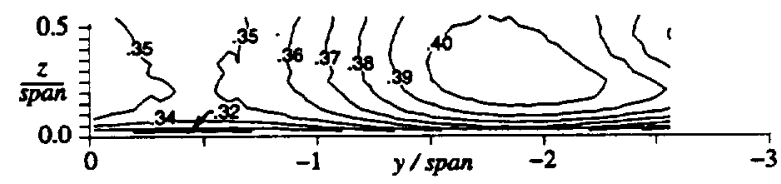

b. Mach number distribution with turbulence grid

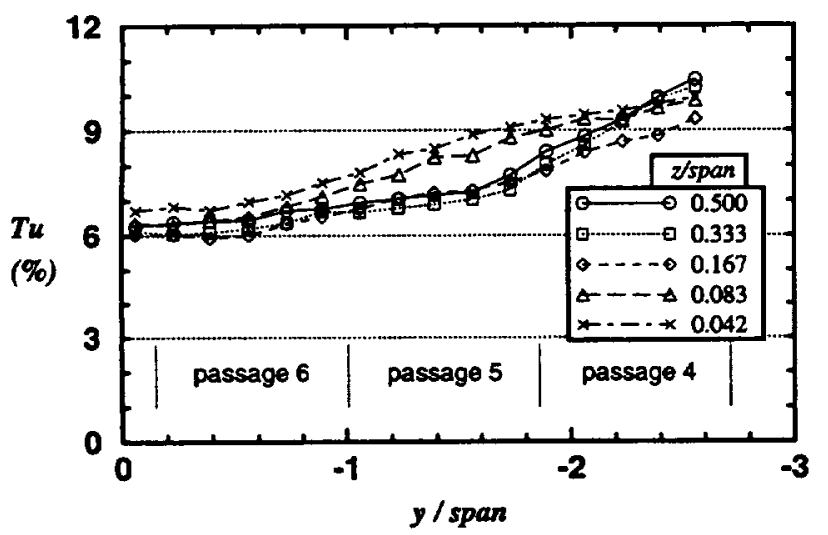

c. turbulence intensity with turbulence grid

Fig. 5 Aerodynamic inlet conditions

Turbulence length scales can have a significant influence on heat transfer rates. A longitudinal, integral turbulence length scale for the present case was estimated by extrapolating data given by Van Fossen et al. (1994) to higher air speeds. Their data was taken in a low speed wind tunnel $(45 \mathrm{~m} / \mathrm{s})$ of the same width $(15.24 \mathrm{~cm})$ and with the same grid configuration (bar size and spacing) as the present test. As shown by Roach (1987) and verified by Van Fossen et al. (1994), the length scale generated by grids is independent of Reynolds number, therefore, extrapolation to higher speeds is reasonable. The length scale, $L_{x} / C_{x}$, thus estimated was 0.76 at the leading edge plane of Passage 5 .

Endwall Static Pressure Measurements As previously discussed, static pressure endwall distributions were needed along with the surface temperatures to evaluate Stanton numbers. Figure 6 shows the static-toinlet total pressure ratio measurements for an exit Mach number of 1.3. Also shown are predictions made using the three-dimensional Navier-Stokes code, RVC3D, (Chima and Yokota, 1990). The analysis was done for flow around a single blade. The graph of the endwall pressure distribution was then offset by one pitch and repeated for comparison with data. Excellent agreement is seen between the data and the calculations with the only substantial difference being with the 0.3 contours downstream of the trailing edge. The minimum $P / P_{\text {in }}^{\prime}$ values for the data and for the calculations were 0.3055 and 0.2796 respectively. These values correspond to only a $0.6^{\circ} \mathrm{C}\left(1^{\circ} \mathrm{F}\right)$ difference in $T_{a w}$. The dots in the experimental data figure show the location of the pressure taps. Even with 87 taps per passage, the experimental data may not have enough resolution to adequately define these low pressure islands. For this reason, the isentropic Mach number used to calculate the Stanton number was obtained from the CFD calculations rather than directly from the data. The calculated Mach number distribution was not affected by a Reynolds number variation, so only two exit Mach number calculations were needed to process the data. Other endwall static pressure data is available from Giel et al. (1996).

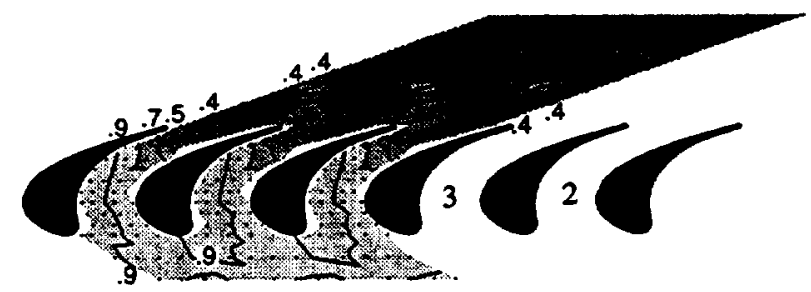

a experimental data with tap locations

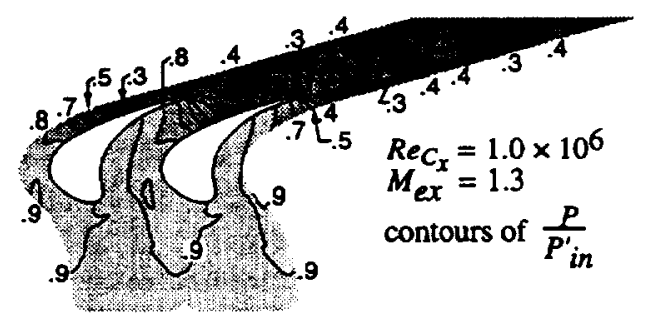

b. CFD calculations

Fig. 6 Endwall static pressure distributions 
Figure 6 also shows the strong three-dimensionality of the flow. This is particularly evidenced by the distortion of the $P / P_{\text {in }}^{\prime}=0.8$ contour line. The distortion is caused by the horseshoe vortex. Pressure measurements on the blade surface, Giel et al. (1996), also showed strong three-dimensionality in the flow field. Near midchord there was nearly a thirty percent decrease in the rotor pressure differential at $6 \%$ of span compared to the midspan value.

Heat Transfer Measurements Heat transfer measurements were made at two Reynolds numbers and at each of two exit Mach numbers with a low turbulence inlet condition. Each of these tests were repeated using the grid to generate a high level of turbulence. Results are presented in this paper for the full factorial experiment of three variables at two levels each, i.e., eight cases. Table 3 lists test conditions for the eight cases. Note that one of the low Reynolds number cases, Case 5, showed a greater variability in exit conditions during the run. However, even this variability was small.

\section{Table 3 Description of endwall heat transfer cases}

\begin{tabular}{|c|l|l|c|}
\hline Case & $R e_{C_{z}}$ & $M_{\text {ex }}$ & $T u$ grid \\
\hline 1 & $0.937 \pm 0.007 \times 10^{6}$ & $1.321 \pm 0.002$ & no \\
2 & $0.938 \pm 0.009 \times 10^{6}$ & $0.962 \pm 0.001$ & no \\
3 & $0.973 \pm 0.016 \times 10^{6}$ & $1.328 \pm 0.001$ & yes \\
4 & $0.952 \pm 0.017 \times 10^{6}$ & $0.992 \pm 0.001$ & yes \\
5 & $0.473 \pm 0.004 \times 10^{6}$ & $1.272 \pm 0.036$ & no \\
6 & $0.475 \pm 0.003 \times 10^{6}$ & $0.961 \pm 0.004$ & no \\
7 & $0.493 \pm 0.003 \times 10^{6}$ & $1.313 \pm 0.006$ & yes \\
8 & $0.493 \pm 0.004 \times 10^{6}$ & $0.982 \pm 0.002$ & yes \\
\hline
\end{tabular}

(all repeatabilities based on $95 \%$ confidence limits)

The endwall Stanton number distributions for the eight cases are presented in Figs. 7 through 14. Each of these figures will be first discussed separately, then compared to each other in order to summarize the effects of Reynolds number, exit Mach number, and the inlet turbulence grid. The figures are arranged such that the effects of Reynolds number can be observed by comparing any two figures that are horizontally opposite each other, e.g., Fig. 7 and Fig. 11. The effects of Mach number can be observed by comparing sequentially numbered figures, e.g., Fig. 7 and Fig. 8. Finally, the effects of the turbulence grid can be observed by comparing every other figure, e.g., Fig. 7 and Fig. 9. Because all of the data was interpolated onto the same rectangular grid, it was possible to subtract the local values from any two cases in order to determine percentage changes in $S t$ brought about by changes in one or more flow parameters. While the authors used this procedure to help interpret the results, the large number of figures generated from this procedure prevents their inclusion here. Simply subtracting $S t$ values at the same $(x, y)$ location from any two figures leads to the same conclusions.

Figure 7 shows the endwall Stanton number distribution for a case with an inlet $R e_{C_{x}}=1.0 \times 10^{6}$, a $P_{\text {ex }} / P_{\text {in }}^{\prime}$ corresponding to an isentropic exit Mach number of 1.3, and without the turbulence grid - Case 1 . The heated section begins $0.3 C_{x}$ upstream of the blade leading edge plane. High Stanton number levels are expected in this region because of the unheated starting length heat transfer effects. Even though the Stanton number is in theory infinite at the heater leading edge, a flat plate correlation (Kays and Crawford, 1980) shows that it is reduced to $2.4 \times 10^{-3}$ at a distance $0.1 C_{x}$ from the start of heating. The low value at this location results from the thick inlet boundary layer which corresponds to a Reynolds number, $R e_{x}$, of $14.6 \times 10^{6}$.

Further downstream, the presence of the blades quickly distorts the Stanton number distribution from the flat plate correlation results. Near the blade leading edge, the Stanton numbers are significantly influenced by the horseshoe vortex structure. As the flow approaches the leading edge, there is a general increase in heat transfer. High Stanton number levels are seen very close to the blade, near its stagnation point, in agreement with the effects observed by Goldstein and Spores (1988) of a leading edge corner vortex. As the flow accelerates around the suction surface, there is a region of very high heat transfer, St $\times 1000>4.5$. In the pitchwise direction, this is a region of high gradients, since near midpassage, there is a relatively low heat transfer region.

A comparison of the data in the primary measurement passage with the data in the adjacent passage indicates that the data shows good periodicity. Minimum heat transfer regions are seen at midchord near each of the pressure surfaces. This is as expected, since as Fig. 6 shows, this is a low velocity region. From here, Stanton number levels gradually increase going downstream. Near the suction surface, levels decrease in the streamwise direction from the peak levels downstream of the stagnation region. The effects of the pressureside leg of the horseshoe vortex are seen clearly further along the suction surface. When the pressure-side leg of the horseshoe vortex lifts off of the endwall, it no longer scours the endwall. A small peak in St of $4.5 \times 10^{-3}$ is observed near the presumed lift-off point. From this point, the passage vortex causes an accumulation of 


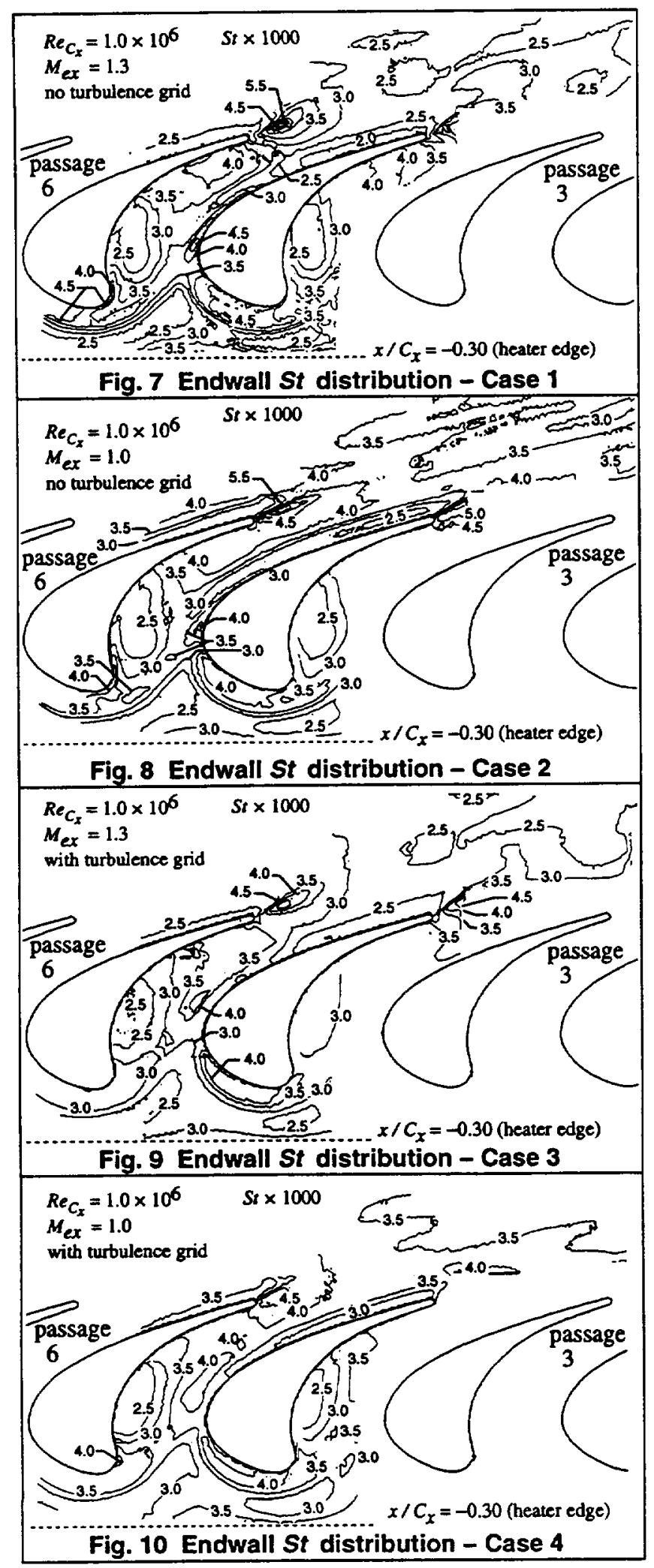

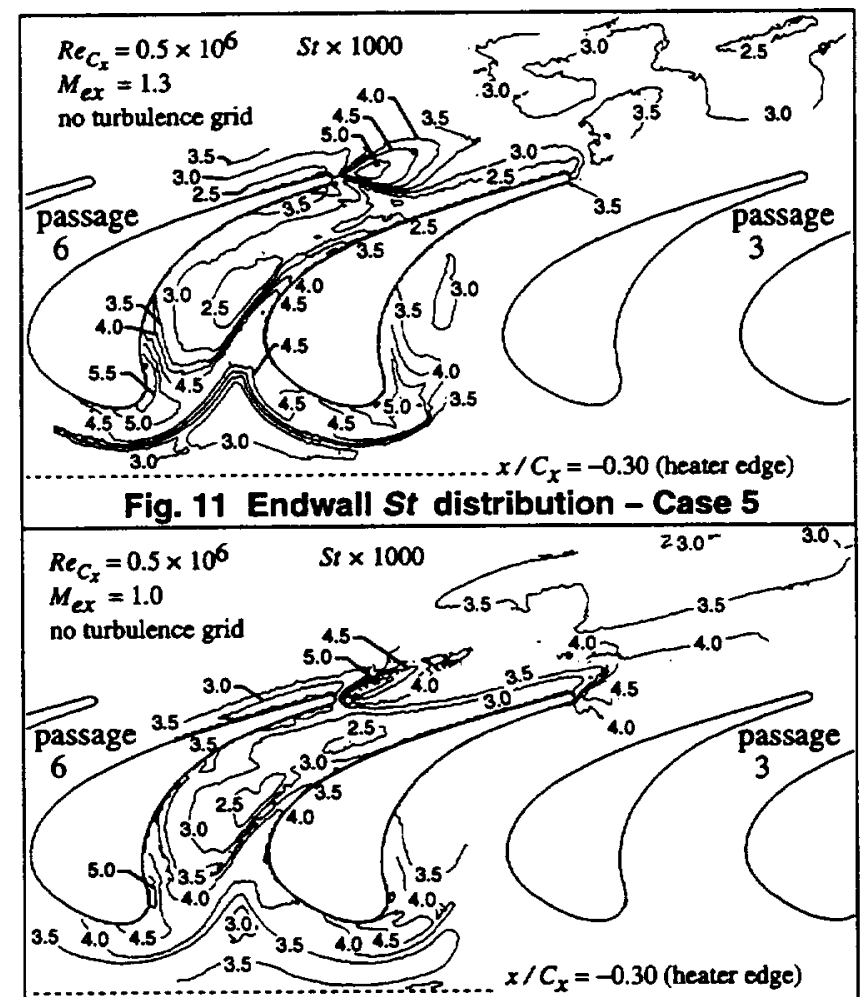

Fig. 12 Endwall St distribution - Case 6

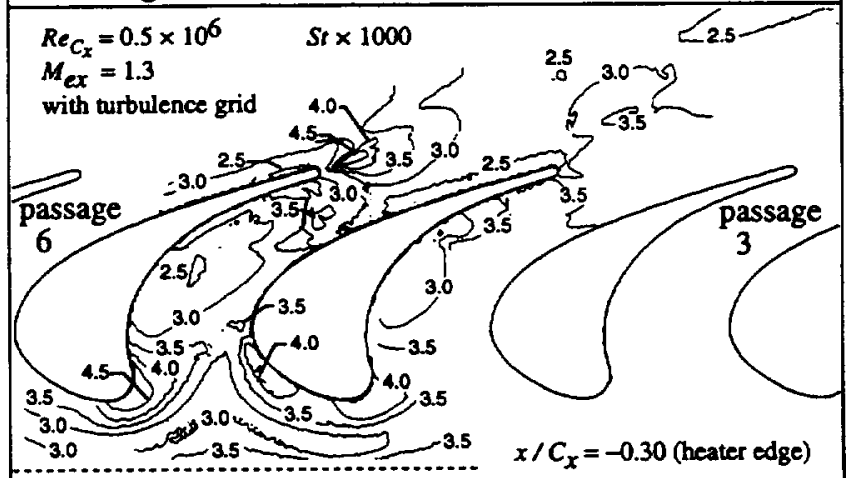

Fig. 13 Endwall St distribution - Case 7

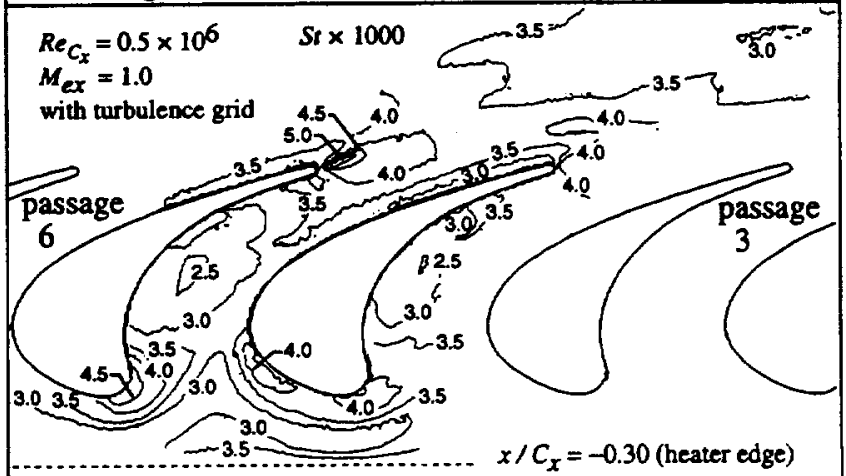

Fig. 14 Endwall St distribution - Case 8 
secondary-flow fluid near the suction surface. This fluid has passed over the heated endwall, thus resulting in lower heat transfer rates near the suction surface. In fact, the lowest Stanton number levels are seen on the endwall downstream of the throat along the uncovered portion of the suction surface. Note that the minimum Stanton number in this region is somewhat lower in the primary measurement passage (Passage 5 ) than in the adjacent passage (Passage 6). This difference is caused by the fact that heaters cover the entire secondary flow path in Passage 5, but only a portion of Passage 6 is covered by heaters. This allows the secondary flow to become warmer in Passage 5 before it reaches the suction surface, thus decreasing the rate of heat transfer.

Highly localized peak endwall heat transfer rates occur in the wake region, 0.20 to $0.25 C_{x}$ downstream of the blade trailing edge. This distance corresponds to approximately 5 to 6 trailing edge diameters. Goldstein and Spores (1988) saw a similar highly localized peak, but for their tests it was much closer to the blade, only 0.06 to $0.07 C_{x}$. downstream of the blade trailing edge. Further downstream of the blade row several distinct regions of low heat transfer are seen. These regions are probably defined by the shock structure evident in Fig. 6. The Stanton number generally decreases in the streamwise direction downstream of the blade row as the thermal boundary layer grows on the endwall. In general, Fig. 7 shows good endwall heat transfer periodicity and strong evidence of the highly three-dimensional flow field.

The endwall Stanton number distribution for Case 2 is shown in Fig. 8. The conditions for this case were the same as for Case 1 except that the isentropic exit Mach number was reduced to slightly less than 1.0 so that no shocks would be present in the downstream region. The mass flows for Case 1 and Case 2 were nearly identical. The endwall heat transfer levels upstream of the throat were expected to be the same as for Case 1. Comparing Figs. 7 and 8 shows that this is generally true within the experimental uncertainty. The figure does not quite show the same peak $S t$ of $4.5 \times 10^{-3}$ near the blade stagnation point as was seen in Case 1. This was probably due to insufficient resolution of $q_{h t r}$ settings when acquiring the data for Case 2. Along the pressure surface, $S t$ increases gradually in streamwise direction with contour lines approximately being normal to the pressure surface. This was also true for Case 1.

Because there are no shocks present to interact with the endwall boundary layers, Stanton number contours from midchord to the exit generally follow the paths of expected inviscid streamlines and do not appear in distinct regions as they did in Case 1 . The low heat transfer region along the uncovered portion of the suction surface is still present but with higher $S t$ levels and with a smaller area. The level of the peak heat transfer $\left(S t>5.5 \times 10^{-3}\right)$ downstream of the trailing edge remains the same, but its location is slightly closer to the trailing edge, approximately 3 to 5 trailing edge diameters downstream. Downstream of the blade row the Stanton number levels are $40 \%$ to $60 \%$ higher than Case 1 and generally follow the blade wakes without being perturbed by shocks.

Figure 9 shows the endwall Stanton number distribution for the same Reynolds number and exit Mach number as Fig. 7 but obtained with the inlet turbulence grid installed. In general, the figure shows, somewhat surprisingly, that the primary effect of the turbulence grid is to lower peak heat transfer levels. The most significant differences are in the regions in front of the blade leading edges, in the path of the horseshoe vortex. The low Stanton number regions of $2.5 \times 10^{-3}$ are still seen upstream of this region. The steep $S t$ gradients near midpitch seen in Fig. 7, however, are missing. Recall from Fig. 5 that the turbulence grid significantly reduced the inlet boundary layer thickness. Blair (1983) showed that, when corrected for the length scale $L_{x}$, a freestream turbulence intensity of $7 \%$ can be expected to result in less than a $10 \%$ increase in St. The reduced secondary flows resulting from the thinner inlet boundary layer may overshadow this level of increased $S t$. The peak $S t$ area seen in Fig. 7 near the presumed vortex lift-off point has moved away from the suction surface, followed by $10 \%$ to $20 \%$ higher Stanton numbers along the remainder of the suction surface. This observation supports the idea that the secondary flow structure is different for the two cases. Figure 9 shows that the region downstream of the blade trailing edge is similar for the two cases. This is probably due to the fact that even for Case 1, the horseshoe vortex structure scours the endwall, increasing the freestream turbulence levels and eliminating the thick inlet boundary layers. Downstream of the horseshoe vortex therefore, the two cases should have similar endwall heat transfer distributions. The far downstream region, in fact, supports this supposition with levels and patterns very similar to those of Case 1.

Figure 10 shows the final high Reynolds number case, Case 4. The trends and differences for this case are generally the same as for the cases discussed above. In comparing Fig. 10 with Fig. 8, it is evident that the increased $S t$ levels near the suction surface are more pronounced for this case. Downstream of the blade row, 
however, the Stanton number levels are again very similar.

Figure 11 shows the endwall Stanton number distribution for Case 5. Contrasting this figure with Fig. 7 shows the effects of reducing the Reynolds number for a supersonic exit case with no inlet turbulence grid. These effects are significant in the upstream and leading edge region. Increases in $S t$ are evident in excess of the 15 to $20 \%$ increase that would be expected assuming that $S t \sim R e^{-0.2}$ based on a turbulent flow analogy. The peak Stanton number in the stagnation region increases from about $4.5 \times 10^{-3}$ for Case 1 to about $5.5 \times 10^{-3}$ for Case 4. Aside from the peak value, the overall shape of the contours in the leading edge region remains about the same, resulting in higher gradients of $S t$ near midpitch. The blade passage, particularly near the pressure surface, shows the most significant Reynolds number effects. The lower Reynolds number moves the minimum $S t$ region significantly closer to the suction surface and slightly downstream. Stanton number levels at quarter-chord near the pressure surface increase by more than $50 \%$. Levels along the suction surface also increase by 10 to $30 \%$. However, levels near midpassage, from 0.5 to $0.75 C_{x}$, decrease by $30 \%$ or more. This all has the effect of producing very large $S t$ gradients near midchord. These large effects are almost certainly due to the thicker inlet boundary layer and the changes in the horseshoe vortex that it brings about. Downstream of the throat, the uncovered suction surface region and, in fact, the entire downstream region appears to show levels that are generally higher than for the $R e_{C_{x}}=1.0 \times 10^{6}$ case. The peak levels downstream of the trailing edge are again nearly the same, but the area of higher heat transfer surrounding this peak is significantly larger.

Figure 12 shows the endwall Stanton number distribution for Case 6. The area upstream of the throat agrees reasonably well with the data of Case 5 . The area downstream of the throat generally shows increased $S t$ levels, the same $M_{e x}$ effect that was seen for other cases. In comparing Fig. 12 with Fig. 8 , it is seen that the Reynolds number effects are also consistent with those discussed above.

The endwall Stanton number distribution for Case 7 is shown in Figure 13. This is a $0.5 \times 10^{6}$ Reynolds number case with the inlet turbulence grid installed. The lower Reynolds number would normally produce a thicker inlet boundary layer, but the turbulence grid reduces it significantly. The net result of these effects is that the low pressure surface $S t$ region characteristic of the higher Reynolds number cases has still moved downstream and closer to the suction surface, but not as much as for the no-grid cases (5 and $6)$. Therefore, the gradients of $S t$ near the suction surface at midchord are not as large as for the no-grid cases. Decreasing the Reynolds number with the turbulence grid installed produces trends similar to the no-grid cases, but the magnitude of the differences is reduced.

The final endwall Stanton number distribution, Case 8, is seen in Fig. 14. Comparing Fig. 14 to Fig. 10 shows the same Reynolds number trends that have been shown throughout. Comparing it to Fig. 12 shows consistent turbulence-grid effects. When compared to Fig. 13, the consistent downstream Mach number trend of $40 \%$ to $60 \%$ higher $S t$ levels associated with the lower $M_{e x}$ levels is again seen. Also, the agreement with Case 7 in the region upstream of the throat is excellent.

\section{SUMMARY AND CONCLUSIONS}

In general, lowering $\operatorname{Re}_{C_{x}}$ by a factor of two has significant effects on the endwall heat transfer in excess of the $15-20 \%$ increases expected from flat plate correlations. Stanton number levels near the pressure surface increase by more than $50 \%$, while levels near the suction surface increase by nearly $30 \%$. Lower Reynolds numbers also move the minimum passage Stanton number slightly downstream and towards the suction surface, decreasing levels at midpassage by $30 \%$ or more. These effects were shown to result in large $S t$ gradients near the suction surface at about midchord. The Reynolds number effects were most evident in Cases 5 and 6 with no grid, but were also seen for Cases 7 and 8 with the turbulence grid.

The shock structure present in the supersonic $M_{\mathrm{ex}}$ cases were shown to break up the smooth $S t$ distributions present in the sonic $M_{e x}$ cases. In general, Stanton number levels are $40 \%$ to $60 \%$ higher for the lower exit Mach number cases. In all cases, the downstream Stanton number levels decrease in the streamwise direction as the thermal boundary layer grows on the endwall.

In general, the data show the somewhat surprising result that the primary effect of the turbulence grid is to lower peak heat transfer levels. This result is thought to be due to the secondary flow change brought about by the reduced inlet boundary layer thickness. Downstream of the blade row the turbulence grid had essentially no effect on the endwall heat transfer.

Overall, the steady-state liquid crystal technique proved to be quite valuable for obtaining CFD code 
validation endwall heat transfer data. The analysis of thermocouple data showed the importance of a careful Stanton number definition. The good spatial resolution offered by the large scale of the cascade and by the liquid crystal technique allowed the effects of the large secondary flows in the blade passages to be clearly quantified. The data show the significant differences in the heat transfer distributions that resulted from variations in Reynolds number, exit Mach number, and inlet turbulence grid. For each of the eight cases, however, a peak heat transfer region occurred in a small downstream region, between 3 to 6 diameters downstream of the trailing edge.

Well-defined inlet flow measurements were presented, important for specifying boundary conditions to CFD codes. The endwall heat transfer data presented here, along with the aerodynamic data presented by Giel et al. (1996) comprise a complete set of data suitable for CFD code and model validation. Also, as required by most CFD analyses, the endwall heat transfer measurements show good periodicity. Electronic tabulations of all eight cases presented in this paper are available upon request. Comparing CFD calculations to all of the eight test conditions available would give greater confidence in the ability of CFD to predict endwall heat transfer.

\section{ACKNOWLEDGEMENTS}

This work was supported by the NASA Lewis Research Center under contract NAS3-27186 with NYMA, Inc., with Mr. Robert J. Boyle as monitor. The support of Ms. Susan Zeleznik in digitizing the data is also gratefully acknowledged.

\section{REFERENCES}

Blair, M. F., 1983, "Influence of Free-Stream Turbulence on Turbulent Boundary Layer Heat Transfer and Mean Profile Development, Part II - Analysis of Results," ASME Journal of Heat Transfer, Vol. 105, pp. 41-47.

Blair, M. F., 1994, "An Experimental Study of Heat Transfer in a Large-Scale Turbine Rotor Passage," ASME Journal of Turbomachinery, Vol. 116, No. 1, pp. 1-13.

Chima, R. V., and Yokota, J. W., 1990, "Numerical Analysis of Three-Dimensional Viscous Internal Flows," AIAA Journal, Vol. 28, No. 5, pp. 798-806.

Eckert, E. R. G., 1955, J. Aero. Sci., pp. 585-587.

Giel, P. W., Sirbaugh, J. R., Lopez, I., and VanFossen, G. J., 1994, “Three-Dimensional Navier-Stokes Analysis and Redesign of an Imbedded Bellmouth Nozzle in a Turbine Cascade Inlet Section," ASME paper 94-GT-237, to appear in the ASME Journal of Turbomachinery.

Giel, P. W., Thurman, D. R., Lopez, I., Boyle, R. J., VanFossen, G. J., Jett, T. J., Camperchioli, W. P., and La, H., 1996, "Three-Dimensional Flow Field Measurements in a Transonic Turbine Cascade," to be presented at the ASME International Gas Turbine Conference, Birmingham, England, June 10-13.

Graziani, R. A., Blair, M. F., Taylor, R. J., and Mayle, R. E., 1980, "An Experimental Study of Endwall and Airfoil Surface Heat Transfer in a Large Scale Turbine Blade Cascade," ASME Journal of Engineering for Power, Vol. 102, No. 2, pp. 1-11.

Gregory-Smith, D. G., and Cleak, J. G. E., 1992, "Secondary Flow Measurements in a Turbine Cascade with High Inlet Turbulence," ASME Journal of Turbomachinery, Vol. 114, No. 1, pp. 173-183.

Gregory-Smith, D. G., Graves, C. P., and Walsh, J. A., 1988, "Growth of Secondary Losses and Vorticity in an Axial Turbine Cascade," ASME Journal of Turbomachinery, Vol. 110, No. 1, pp. 1-8.

Gregory-Smith, D. G., and Graves, C. P., 1983, "Secondary Flows and Losses in a Turbine Cascade," in: Viscous Effects in Turbomachines, AGARD-CP-351.

Goldstein, R. J., and Spores, R. A., 1988, "Turbulent Transport on the Endwall in the Region Between Adjacent Turbine Blades," ASME Journal of Heat Transfer, Vol. 110, No. 4, pp. 862-869.

Hippensteele, S. A., and Russell, L. M., 1988, "High-Resolution Liquid Crystal Heat-Transfer Measurements on the End Wall of a Turbine Passage With Variations in Reynolds Number," presented at the 25th ASME Heat Transfer Conf., Houston, Texas, July 2427, also NASA TM-100827. 
Kays, W. M., and Crawford, M. E., 1980, Convective Heat and Mass Transfer, 2nd. Ed., McGraw-Hill, New York.

Kline, S. J., and McClintock, F. A., 1953, "Describing Uncertainty in Single-Sample Experiments," Mechanical Engineering, Vol. 75, Jan., pp. 3-8.

Langston, L. S., Nice, M. L., and Hooper, R. M., 1977, "Three Dimensional Flow Within a Turbine Cascade Passage," ASME Journal of Engineering for Power, Vol. 99, No. 1, pp. 21-28.

Marchal, P., and Sieverding, C. H., 1977, "Secondary Flows Within Turbomachinery Bladings," in: Secondary Flows Within Turbomachines, AGARD-CP214.

Martinez-Botas, R. F., Lock, G. D., and Jones, T. V., 1994, "Heat Transfer Measurements in an Annular Cascade of Transonic Gas Turbine Blades Using the Transient Liquid Crystal Technique," ASME 94GT-172.

Mee, D. J., Baines, N. C., Oldfield, M. L. G., and Dickens, T. E., 1992a, "An Examination of the Contributions to Loss on a Transonic Turbine Blade in Cascade," ASME Journal of Turbomachinery, Vol. 114, No. 1, pp. 155-162.

Mee, D. J., Baines, N. C., and Oldfield, M. L. G., 1992b, "Detailed Boundary Layer Measurements on a Transonic Turbine Cascade," ASME Journal of Turbomachinery, Vol. 114, No. 1, pp. 163-172.

Moffat, R. J., 1990, "Experimental Heat Transfer," Proc. of the Ninth Int'l Heat Transfer Conf., Jerusalem, Israel, Vol. 1, pp. 187-205.

Roach, P. E., 1987, "The Generation of Nearly Isotropic Turbulence by Means of Grids," Int. J. Heat and Fluid Flow, Vol. 8, pp. 82-92.

Van Fossen, G. J., Simoneau, R. J., and Ching, C. Y., 1994, "Influence of Turbulence Parameters, Reynolds Number, and Body Shape on StagnationRegion Heat Transfer," NASA TP-3487.

Verhoff, V. G., Camperchioli, W. P., and Lopez, I., 1992, "Transonic Turbine Blade Cascade Testing Facility," AIAA Paper No. 92-4034, NASA TM-105646.

Yamamoto, A., 1987a, "Prediction and Development of Secondary Flows and Losses in Two Types of Straight Turbine Cascades: Part 1 - A Stator Case," ASME Journal of Turbomachinery, Vol. 109, No. 2, pp. 186-193.

Yamamoto, A., 1987b, "Prediction and Development of Secondary Flows and Losses in Two Types of Straight Turbine Cascades: Part 2 - A Rotor Case," ASME Journal of Turbomachinery, Vol. 109, No. 2, pp. 194-200. 
Public reporting burden for this collection of information is estimated to average 1 hour per response, Including the time lor revlewing instructions, searching exialing data sources, gathering and maintaining the data needed, and completing and reviewing the collection of information. Send comments regarding thts burden eavima of any outen appect of this Davis Highway, Suite 1204, Aftington, VA 22202-4302, and to the Otfice of Management and Budgel, Paperwork Reduction Projact (0704-0189), Washington, DC 20503.

\begin{tabular}{|l|l|l|l|}
\hline 1. AGENCY USE ONLY (Leave blank) & $\begin{array}{c}\text { 2. REPOAT DATE } \\
\text { December } 1996\end{array}$ & $\begin{array}{r}\text { 3. REPORT TYPE AND DATES COVERED } \\
\text { Technical Memorandum }\end{array}$ \\
\hline
\end{tabular}

\section{TITLE AND SUBTITLE}

Endwall Heat Transfer Measurements in a Transonic Turbine Cascade

6. AUTHOR(S)
5. FUNDING NUMBERS

WU-523-26-13

P.W. Giel, D.R. Thurman, G.J. Van Fossen, S.A. Hippensteele, and R.J. Boyle

7. PERFOAMNG ORGANIZATION NAME(S) AND ADDRESS(ES)

NASA Lewis Research Center

Cleveland, Ohio 44135-3191

and

U.S. Amy Research Laboratory

Cleveland, Ohio 44135-3191

National Aeronautics and Space Administration

Washington, DC 20546-0001

and

U.S. Army Research Laboratory

Adelphi, Maryland 20783-1145

8. PERFORMNG ORGANIZATION

REPORT NUMBER

E-10583

10. SPONSORINGMONITORING AGENCY REPORT NUMBER

NASA TM-107387

ARL-TR-1253

\section{SUPPLEMENTARY NOTES}

Prepared for the 41 st Gas Turbine and Aeroengine Congress sponsored by the International Gas Turbine Institute of the American Society of Mechanical Engineers, Birmingham, United Kingdom, June 10-13, 1996. P.W. Giel, NYMA, Inc., 2001 Aerospace Parkway, Brook Park, Ohio 44142 (work funded under NASA Contract NAS3-27186); D.R. Thurman, U.S. Ammy Research Laboratory, NASA Lewis Research Center; G.J. Van Fossen, S.A. Hippensteele, and R.J. Boyle, NASA Lewis Research Center. Responsible person, Robert J. Boyle, organization code 5820, (216) 433-5889.

12a. DISTRIBUTIONAVAILABILITY STATEMENT 12b. DISTRIBUTION CODE

Unclassified - Unlimited

Subject Categories 02 and 07

This publication is available from the NASA Center for AeroSpace Information, (301) 621-0390.

13. ABSTRACT (Maximum 200 words)

Turbine blade endwall heat transfer measurements are given for a range of Reynolds and Mach numbers. Data were obtained for Reynolds numbers based on inlet conditions of 0.5 and $1.0 \times 10^{6}$, for isentropic exit Mach numbers of 1.0 and 1.3 , and for freestream turbulence intensities of $0.25 \%$ and $7.0 \%$. Tests were conducted in a linear cascade at the NASA Lewis Transonic Turbine Blade Cascade Facility. The test article was a turbine rotor with $136^{\circ}$ of tuming and an axial chord of $12.7 \mathrm{~cm}$. The large scale allowed for very detailed measurements of both flow field and surface phenomena. The intent of the work is to provide benchmark quality data for CFD code and model verification. The flow field in the cascade is highly three-dimensional as a result of thick boundary layers at the test section inlet. Endwall heat transfer data were obtained using a steady-state liquid crystal technique.

14. SUBJECT TERMS

Gas turbines; Heat transfer; Cascade wind tunnel; Transonic flow; Supersonic turbines

\begin{tabular}{|c|c|}
\hline $\begin{array}{c}\text { 17. SECURTY CLASSIFICATION } \\
\text { OF REPORT }\end{array}$ & $\begin{array}{c}\text { 18. SECURTY CLASSIFICATION } \\
\text { OF THIS PAGE } \\
\text { Unclassified }\end{array}$ \\
Unclassified \\
\hline
\end{tabular}

19. SECURITY CLASSIFICATION OF ABSTRACT

Unclassified 